\title{
The Zinc Transporter SLC39A7 (ZIP7) Is Essential for Regulation of Cytosolic Zinc Levels $\$$
}

\author{
Grace Woodruff, Christian G. Bouwkamp, Femke M. de Vrij, Timothy Lovenberg, \\ Pascal Bonaventure, Steven A. Kushner, and Anthony W. Harrington
}

Neuroscience Discovery, Janssen Research and Development, San Diego, California (G.W., T.L., P.B., A.W.H.); and Department of Psychiatry, Erasmus MC, Rotterdam, The Netherlands (C.G.B., F.M.V., S.A.K.)

Received March 22, 2018; accepted June 28, 2018

\section{ABSTRACT}

Zinc homeostasis is a highly regulated process in mammalian cells that is critical for normal growth and development. Movement of zinc across cell compartments is controlled by two classes of transporters: Slc39a family members transport zinc into the cytosol from either the extracellular space or intracellular stores such as the endoplasmic reticulum (ER), whereas the SLC30A family mediates zinc efflux from the cytosol. In this study, we report that genetic ablation of SLC39A7 (ZIP7) results in decreased cytosolic zinc levels, increased ER zinc levels, impaired cell proliferation, and induction of ER stress. Confirmatory of impaired zinc transport as the causal mechanism, both the increased ER stress and impaired cell proliferation were rescued by increasing cytosolic zinc. Furthermore, using these robust cellular phenotypes, we implemented a small-molecule library screen with 2800 compounds and identified one small molecule capable of rescuing ER stress and cell proliferation in ZIP7-deficient cells in the low micromolar range.

\section{Introduction}

Zinc is an essential metal that is required for normal growth and development in all higher plants and animals. Acting as a cofactor for over 2000 proteins, zinc can modulate enzymatic activity and gene transcription (Hogstrand et al., 2009), as well as function as a second messenger in signaling pathways (Yamasaki et al., 2007). Because zinc cannot freely permeate lipid bilayers, active transport is required to move zinc across cellular and organelle membranes. There are two families of zinc transporters, categorized by their direction of zinc movement. The solute carrier family 30A (SLC30A), also known as the $\mathrm{Zn}$ transporter (ZnT) family, has 10 members that mobilize zinc from the cytosol. The solute carrier family 39A (SLC39A) family, also known as the Zrt-, Irt-related protein (ZIP) family, has 14 members that transport zinc into the cytosol from the extracellular space or from intracellular stores, such as the ER and Golgi. In addition to zinc, some members of the ZIP family also transport iron (Pinilla-Tenas et al., 2011), manganese (Girijashanker et al., 2008), and cadmium (Fujishiro et al., 2012).

Recent publications have identified the importance of $\mathrm{ZnT}$ and ZIP transporters in human health and disease. Mutations in SLC39A8 cause intellectual disability with cortical atrophy (Boycott et al., 2015); mutations in SLC39A14 (ZIP14) cause

https://doi.org/10.1124/mol.118.112557.

S This article has supplemental material available at molpharm. aspetjournals.org. childhood-onset parkinsonism-dystonia (Tuschl et al., 2016); and mutations in SLC39A13 (ZIP13) cause a form of EhlersDanlos syndrome (Giunta et al., 2008). Additionally, singlenucleotide polymorphisms and copy number variations in other ZnT and ZIP family members have been associated with bipolar disorder (Baum et al., 2008), schizophrenia (Carrera et al., 2012), autism (O'Roak et al., 2011; Gazzellone et al., 2014), and other disorders (Kambe et al., 2015). Thus, elucidating the function of individual zinc transporters may help us understand the pathophysiology, treatment, and prevention of zinc-related disease conditions.

ZIP7 is the only known ZIP family member that is localized to the ER membrane and is hypothesized to be responsible for mobilizing zinc from the ER, a zinc storage site, into the cytosol (Taylor et al., 2004). ZIP7 is critical for normal growth and development as the ZIP7 knockout (KO) is embryonic lethal in mice, whereas loss-of-function mutations of the drosophila ZIP7 homolog Catecholamines up (Catsup) lead to abnormal wing development (Groth et al., 2013). Furthermore, morpholino knockdown of ZIP7 expression in zebrafish causes neurodevelopmental impairments, which can be rescued with zinc supplementation (Yan et al., 2012). At the cellular level, recent studies have illuminated a role for ZIP7 in the normal function of the ER and shown that loss of ZIP7 results in ER stress (Ohashi et al., 2016; Bin et al., 2017). However, the precise mechanism of how loss of ZIP7 causes ER stress and affects zinc levels has not been fully elucidated. In this work, we report that clustered regularly interspaced short palindromic repeats

ABBREVIATIONS: CHOP, CCAAT enhancer-binding protein homologous protein; CRISPR, clustered regularly interspaced short palindromic repeats; ER, endoplasmic reticulum; GAPDH, glyceraldehyde-3-phosphate dehydrogenase; GFP, green fluorescent protein; GO, Gene Ontology; ICP-MS, inductively coupled plasma mass spectrometry; KO, knockout; PBS, phosphate-buffered saline; PCR, polymerase chain reaction; PDI, protein disulfide isomerase; RNA-seq, RNA sequencing; WT, wild-type; ZIP, Zrt-, Irt-related protein; ZnT, Zn transporter. 
(CRISPR)-mediated genetic ablation of ZIP7 in human cells results in severely decreased levels of cytosolic zinc, impaired cell proliferation, and activation of ER stress. Moreover, we demonstrate the causality of the impaired cytosolic zinc levels by fully rescuing both the impairment of cell proliferation and activation of ER stress by restoring cytosolic zinc levels. Finally, we implemented a small-molecule screen based on the ER stress phenotype that yielded a lead compound with efficacy in fully rescuing the effects of ZIP7 KO through a zinc-independent mechanism.

\section{Materials and Methods}

Cell Culture and Genome Editing. MG-63 cells were grown in Eagle's minimum essential media supplemented with $10 \%$ fetal bovine serum, $1 \times$ Pen/Strep, $1 \times$ non-essential amino acids, and $1 \times$ glutamine. Cells were split with trypsin every 3 to 4 days. MG-63 cells were transfected with Lipofectamine 3000 (Thermofisher, Carlsbad, CA) and SpCas9 plasmid with single guide RNA sequence: GCCACACTCACGAGAGCATCTGG. This single guide RNA sequence targets exon 1 of SLC39A7. Seventy-two hours after transfection, green fluorescent protein (GFP)-positive cells were sorted and plated at a density of 5000 cells per $10-\mathrm{cm}$ dish, such that single colonies could be isolated. Clones were manually picked when colonies were $\sim 100$ cells. Colonies were expanded and screened for ZIP7 KO based on disruption of DNA sequence and confirmed as KO by Western blot. Cell lines were routinely checked to be free of mycoplasma infection using Lonza MycoAlert Mycoplasma Detection Kit (LT07; Lonza, Basel, Switzerland).

Western Blot. Cells were lysed using radioimmunoprecipitation assay buffer (ThermoFisher) with protease (Sigma-Aldrich, St. Louis, MO) and phosphatase (Pierce, Waltham, MA) inhibitors. After centrifugation at $13,000 \mathrm{~g}$, protein concentration was measured using the bicinchoninic acid protein assay kit (Pierce) and lysates were separated on 4\%-12\% Bis-Tris gels (Invitrogen, Carlsbad, CA) using 4-morpholineethanesulfonic acid sodium dodecyl sulfate running buffer (Invitrogen). Proteins were transferred with the iBlot system onto nitrocellulose membranes (Novex, Thermofisher). Membranes were blocked with 5\% milk (Sigma-Aldrich) and then incubated with primary antibodies overnight at $4^{\circ} \mathrm{C}$. Primary antibodies used were as follows: SLC39A7 anti-rabbit (1:500, 117560; Abcam, Cambridge, UK) and glyceraldehyde-3-phosphate dehydrogenase (GAPDH) anti-mouse (1:1000, 374; EMD Millipore, Burlington, MA). Blots were visualized using enhanced chemiluminescence Western blotting detection reagents (Pierce).

Immunocytochemistry and Image Analysis. In each experiment, cells were plated at a density of 1500 per well in 384-well Cell Carrier Ultra microplates (PerkinElmer, Waltham, MA). Cells were fixed with $4 \%$ paraformaldehyde at room temperature for 15 minutes, followed by three washes with phosphate-buffered saline (PBS). Cells were blocked in blocking buffer [5\% BSA (Sigma-Aldrich) with $0.1 \%$ Triton-X (Sigma-Aldrich) in PBS] for 1 hour at room temperature, followed by primary antibody incubation in blocking buffer overnight at $4^{\circ} \mathrm{C}$. Cells were washed three times with PBS and then incubated with secondary antibodies for 1 hour at room temperature diluted in blocking buffer. Cells were washed three times with PBS and then imaged on a PerkinElmer Opera Phenix. Primary antibodies used were as follows: protein disulfide isomerase (PDI) anti-mouse (1:1000, 2792; Abcam), PDI anti-rabbit (1:1000, C81H6; Cell Signaling, Danvers, MA), SLC39A7 anti-rabbit (1:500, 117560; Abcam), and CCAAT enhancer-binding protein homologous protein (CHOP) antimouse (1:3000, L63F7; Cell Signaling). HCS CellMask Deep Red Stain (ThermoFisher) was used during the secondary antibody incubation at $1: 10,000$ to identify the entire cell. To stain nuclei, NucBlue (ThermoFisher) was used during the secondary antibody incubation per the manufacturer instructions. Secondary antibodies used were as follows: goat anti-rabbit Alexa Flour 488, goat anti-mouse Alexa Flour 488, and goat anti-rabbit Alexa Flour 568 all from ThermoFisher were used at 1:500. In every experiment, five images per well were captured at $20 \times$ magnification, and each experiment was performed in triplicate and repeated a minimum of three times. Images were analyzed using Harmony Image Analysis System (PerkinElmer). Please see the Supplemental Data for more detail on image analysis. GraphPad Prism version 7 was used to perform all statistical analysis. All statistical tests were unpaired.

Phenotypic Screen. ZIP7 ${ }^{-1-}$ cells were plated at a density of 1500 cells/well into 384-well Cell Carrier Ultra microplates (PerkinElmer). Twenty-four hours after plating, cells were treated with $1 \mu \mathrm{M}$ compound. Pyrithione was used as the positive control at $500 \mathrm{nM}$. Cells were fixed with $4 \%$ paraformaldehyde 48 hours after compound treatment. In the primary screen, cells were stained with CHOP and HCS CellMask Deep Red Stain, as described above, and the CHOP nucleus/cytoplasm ratio was calculated using Columbus Image Analysis system. Hits were selected based on percentage of inhibition of CHOP. Pyrithione was set as $100 \%$ inhibition, and hits were determined based on $70 \%$ inhibition or above. In the secondary screen, the 33 hits from the primary screen were retested at $1 \mu \mathrm{M}$ in triplicate for 48 hours. In the secondary screen, cells were stained with CHOP, HCS Cell Mask, and PDI, as described above. Of the 33 primary hits, 30 of them confirmed to inhibit CHOP in the secondary screen. One compound (compound A) was identified to not only inhibit CHOP, but also increased cell number and reduced PDI intensity and area.

Synthesis of Compound A. The synthesis of compound A is described in U.S. Patent US2008/080081 in example 24 (Illig et al., 2016).

Zinc Quantification by Inductively Coupled Plasma Mass Spectrometry. Cells were plated in $10-\mathrm{cm}$ dishes and were harvested 72 hours after plating. Dishes were treated with either vehicle $\left(\mathrm{H}_{2} \mathrm{O}\right)$ or $500 \mathrm{nM}$ pyrithione. Equal number of cells was fractionated to cytosol and ER fractions by first collecting cells and spinning down at $300 \mathrm{~g}$. Supernatant was removed, and cells were resuspended in sucrose buffer (0.32 $\mathrm{M}$ sucrose $+10 \mathrm{mM}$ HEPES). Cells were homogenized by syringe-based homogenization. Next, homogenized cells were spun at $300 \mathrm{~g}$ for 10 minutes to remove nuclei. Supernatant from homogenized cells was then centrifuged at $100,000 \mathrm{~g}$ for 1 hour. The supernatant was removed and analyzed as the cytosolic fraction. The pellet was resuspended in sucrose buffer and analyzed as the microsome fraction that contains the ER. Zinc concentrations in the cytosol and microsome fraction were determined by inductively coupled plasma mass spectrometry (ICP-MS). Iridium was used as the internal standard. Samples or standards were combined with the internal standard, digested in $1 \%$ nitric acid, and then infused into the ICP-MS for quantitation. Zinc concentrations were normalized to the total number of cells.

RNA-Seq and Quantitative Polymerase Chain Reaction. Total RNA from three biologic replicates per genotype was extracted using the Qiagen RNeasy Mini Kit, according to the manufacturer's instructions. RNA sequencing (RNA-seq) and analysis were performed by Novogene (Beijing, China). Briefly, libraries were constructed using NEBNext UltraTM RNA Library Prep Kit following manufacturer's instructions, and polymerase chain reaction (PCR) products were purified using AMPure XP System (Beckman Coulter, Brea, CA). The library quality was assessed on the Agilent Bioanlyzer 2100 system. The library was sequenced on an Illumina Hiseq platform. On average, 65.8 million reads were obtained per sample. Raw reads were processed through Novogene in-house scripts to obtain clean reads, by removing reads containing adapters, reads containing poly-N and low-quality reads. On average, 53 million clean reads were obtained per sample. Reads were aligned to the reference genome using TopHat v2.0.12; $89.84 \%$ of reads were uniquely mapped. Fragments per kilobase of transcript per million mapped reads of each gene were calculated based on the length of the gene and reads mapped to each gene. Differential gene expression analysis was performed using DESeq2 $\mathrm{R}$ package. The $P$ values were adjusted 

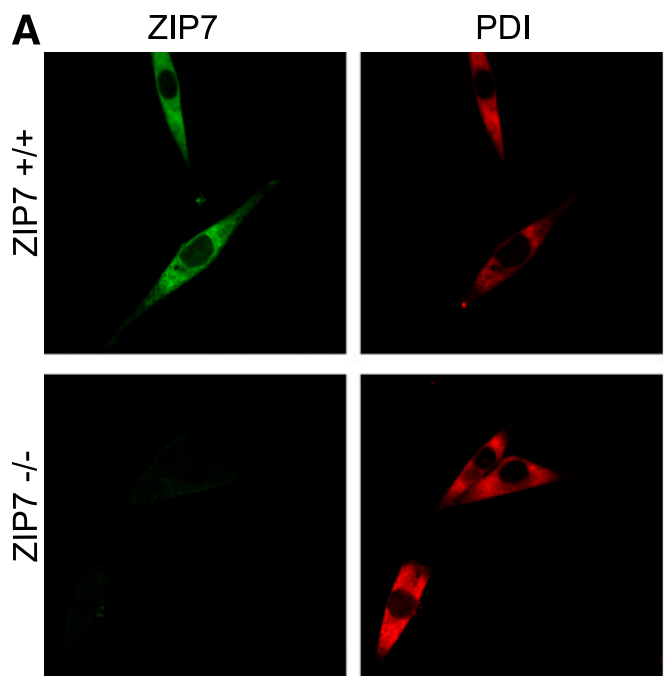

C
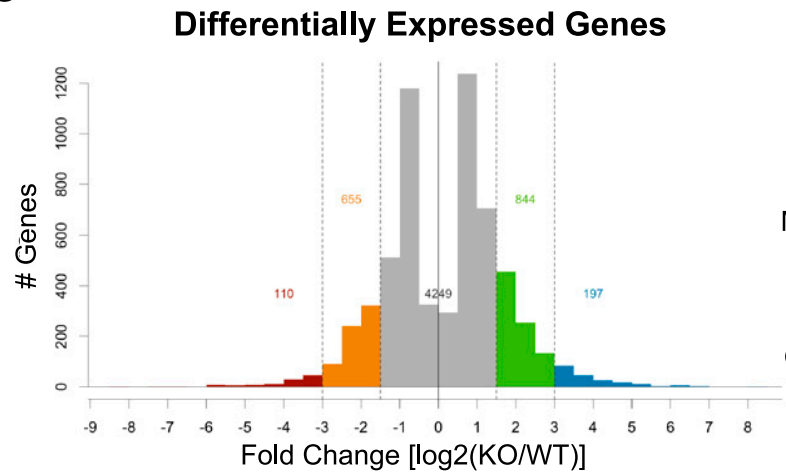
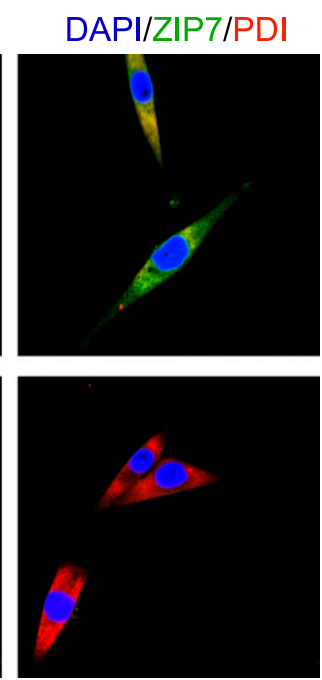

E

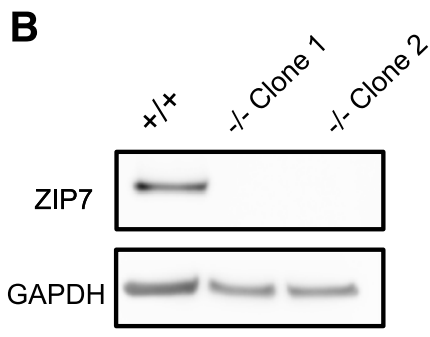

D

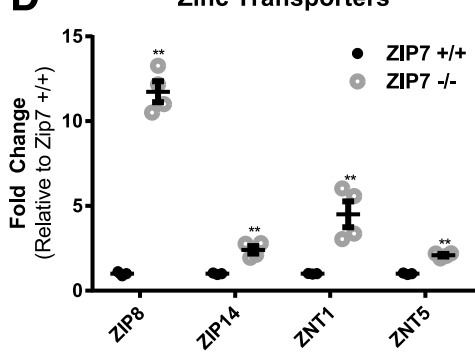

Gene Ontology Enrichment

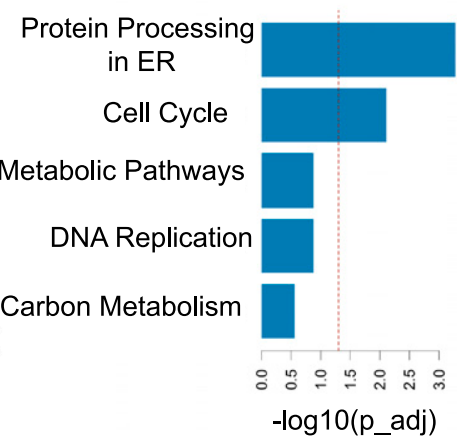

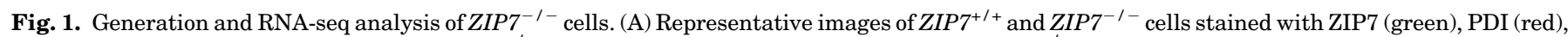

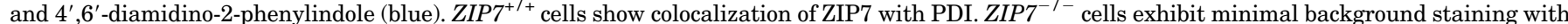

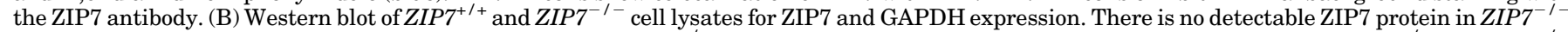

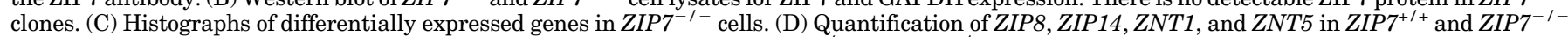

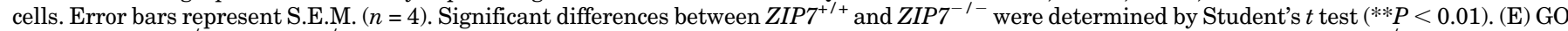

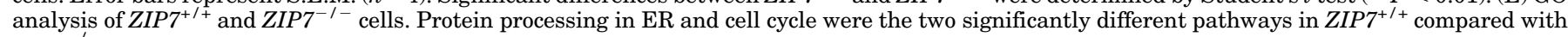
$\mathrm{ZIP}^{-1-}$

using Benjamini and Hochberg's method. Genes with an adjusted $P$ value $<0.05$ were considered as differentially expressed. Gene Ontology (GO) enrichment analysis was performed using GOseq, based on Wallenius noncentral hypergeometric distribution.

For quantitative PCR, total RNA was extracted as described above. RNA was reverse transcribed into cDNA using Superscript III reverse transcriptase (Invitrogen) with random hexamer primers. Transcript abundance was determined by quantitative PCR using SYBR Green PCR mix (Applied Biosystems, Foster City, CA). Transcripts were normalized to GAPDH, and each sample was run in triplicate with three biologic samples per genotype. SLC39A8 (ZIP8) forward, GGCCCCTTCAAACAGGTACA and reverse, TGCTGTCACAGAAGCTAATGG; SLC39A14 (ZIP14) forward, GCAGCTTCATGGTGACTGAA and reverse, GCTAAGCTGCTTCTGCCG SLC30A1; ZNT1 forward, TCACCACTTCTGGGGTTTTC and reverse, ACCAGGAGGAGACCAACACC SLC30A5; ZNT5 forward, TTTGAAGGCTGTGGGACTTTTCG and reverse, GGTGTTTGGTAATAGTTTTCCCAG; GAPDH forward, AGGTCGGTGTGAACGGATTTG and reverse, TGTAGACCATGTAGTTGAGGTCA.

\section{Results}

Generation and RNA-seq Analysis of ZIP7 KO Cells. To elucidate the role of ZIP7 in human cells, we used CRISPR genome editing (Shalem et al., 2014) to genetically ablate ZIP7 from the osteosarcoma cell line, MG-63. MG-63 cells are large cells that are ideal for imaging and amendable to transfection and viral transduction. We identified two clones of 18 screened that had indels in the first exon that introduce premature stop codons. ZIP7 mRNA was significantly decreased in $Z I P 7^{-/-}$ cells, consistent with nonsense-mediated decay. Moreover, ZIP7 protein was undetectable by Western blot in $Z I P 7^{-1-}$ clones (Fig. 1B). We next used $Z I P 7^{+/+}$and $Z I P 7^{-/-}$cells to confirm the localization of ZIP7 to the ER, as has previously been reported (Taylor et al., 2004; Bin et al., 2017). Immunocytochemical labeling of $\mathrm{ZIP}^{+/+}$cells with antibodies to the ER-specific enzyme protein disulfide isomerase (PDI) and ZIP7 confirmed strong colocalization of ZIP7 with PDI, validating the localization of ZIP7 to the ER (Fig. 1A). Importantly, ZIP7 staining was absent other than minimal background labeling in $\mathrm{ZIP} 7^{-/-}$cells, thereby confirming the specificity of the ZIP7 antibody.

To investigate the function of ZIP7 in human cells, we performed RNA-seq on $Z I P 7^{+/+}$and $Z I P 7^{-/-}$cells. There were 6055 differentially expressed genes between $Z I P 7^{+/+}$ and $Z I P 7^{-1-}$ cells with an adjusted $P$ value of $<0.05$ (Fig. 1C), including upregulation of four zinc transporters. 
A

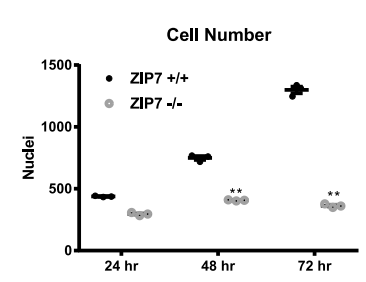

B

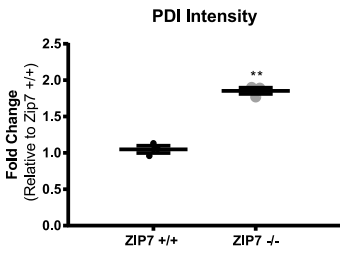

D

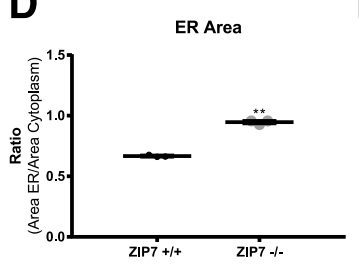

E

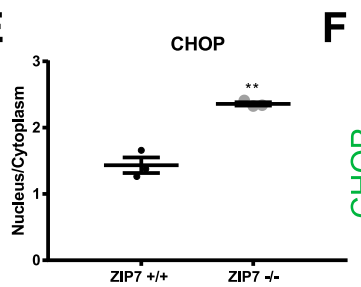

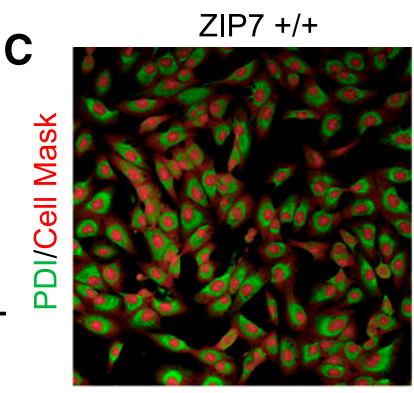
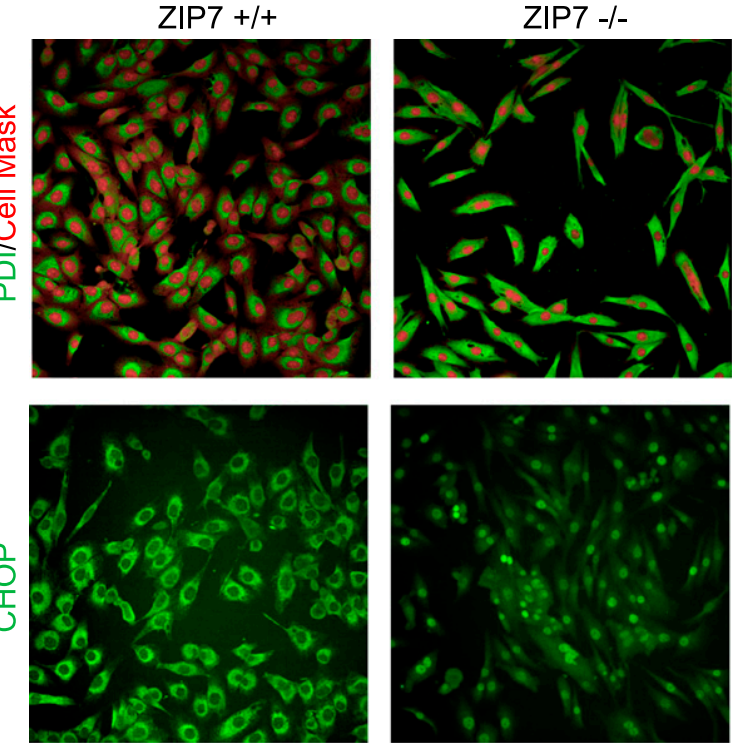

Fig. 2. $Z I P 7 \mathrm{KO}$ causes decreased proliferation and induction of ER stress. (A) Quantification of cell number in $Z I P 7^{+/+}$and $Z I P 7^{-/-}$cells after 24,48 , and 72 hours. There are significantly less $Z I P 7^{-1-}$ cells compared with $Z I P 7^{+/+}$cells, 48 and 72 hours after plating. (B) Quantification of PDI intensity in $Z I P 7^{+/+}$and $Z I P 7^{-1-}$ cells. There is a significant increase in PDI intensity in $Z I P 7^{-\prime-}$ relative to $Z I P 7^{+/+}$cells. Values are expressed as a fold change in intensity relative to $Z I P 7^{+/+}$cells. (C) Representative images of $Z I P 7^{+/+}$and $Z I P 7^{-/-}$cells. PDI (green) and CellMask (red). (D) Quantification of ER area in $Z I P 7^{+/+}$and $Z I P 7^{-/-}$cells. There is a significant increase in the ER area in $Z I P 7^{-1-}$ cells compared with $Z I P 7^{+/+}$cells. (E) Quantification of CHOP in the nucleus versus the cytoplasm in $Z I P 7^{+{ }^{+}}$and $Z I P 7^{-\prime-}$ cells. There is a significant increase in CHOP in the nucleus versus the cytoplasm in $Z I P 7^{-\prime-}$ cells compared with $Z I P 7^{+I^{+}}$cells. (F) Representative images of $Z I P 7^{+/+}$and $Z I P 7^{-1-}$ cells stained with CHOP (green). Error bars represent S.E.M. from three independent experiments $(n=3)$. Significant differences between $Z I P 7^{+/+}$and $Z I P 7^{-{ }^{-}}$cells were determined by Student's $t$ test $(* * P<0.01$ ).

The transporters ZIP8, ZIP14, ZNT1, and ZNT5 all had a twofold or greater upregulation in $Z I P 7^{-/-}$versus $Z I P 7^{+/+}$ cells when measured by quantitative-PCR analysis (Fig. 1D). To further identify cellular processes that were altered by deletion of ZIP7, we performed GO analysis. GO analysis revealed two significantly enriched categories, as follows: 1) protein processing in the endoplasmic reticulum and 2) cell cycle (Fig. 1E). These results are consistent with a recent report that $Z I P 7$ knockdown induces ER stress genes and downregulates genes involved in the cell cycle (Bin et al., 2017).

Loss of ZIP7 Causes Decreased Cell Proliferation and ER Stress. To establish the RNA-seq hypothesized functional consequences of ZIP7 $\mathrm{KO}$, we first examined cell proliferation. We plated equal numbers of $Z I P 7^{+/+}$and $Z I P 7^{-1-}$ cells and quantified the number of nuclei at 24,48 , and 72 hours. $Z I P 7^{+/+}$cells more than doubled in number during the 72-hour period, whereas the number of $Z I P 7^{-/-}$ cells did not significantly increase (Fig. 2A). We next examined whether $Z I P 7^{-1-}$ cells exhibit ER stress as predicted by the RNA-seq data by comparing the staining intensity of the ER marker PDI in $Z I P 7^{+/+}$and $Z I P 7^{-1-}$ cells (Fig. 2C). Indeed, $Z I P 7^{-1-}$ cells exhibited significantly increased intensity of PDI (Fig. 2B), consistent with ER stress (Ko et al., 2002). Moreover, in addition to the increase of PDI intensity, we also observed a parallel increase of the area of PDI staining in $Z I P 7^{-1-}$ cells. Specifically, we quantified the area of PDI staining as a proportion of the cytoplasmic area, which confirmed a significant expansion of the PDI-labeled ER in $Z I P 7^{-1-}$ cells (Fig. 2D), again consistent with ER stress. To further evaluate this possibility, we also examined the localization of CHOP, a transcription factor that is localized to the cytoplasm under normal conditions, but translocates to the nucleus under conditions of ER stress (Ron and Habener, 1992).
Consistent with ER stress, ZIP7 ${ }^{-1-}$ cells exhibited strong nuclear localization of CHOP in contrast to $Z I P 7^{+/+}$cells (Fig. 2, E and F). Taken together, based on the increased intensity of PDI labeling, expansion of PDI-labeled ER area, and nuclear localization of CHOP, there is substantial evidence to suggest that $Z I P 7^{-/-}$cells have increased ER stress.

Wild-Type ZIP7 Rescues Proliferation and ER Stress Phenotypes. To confirm that both the cell proliferation and ER stress phenotypes are dependent on ZIP7, we infected cells with lentivirus to overexpress either GFP, wild-type (WT) ZIP7, or a previously reported loss-of-function ZIP7 ${ }^{\mathrm{G} 178 \mathrm{D}}$ mutant (Groth et al., 2013). Overexpression of WT ZIP7 in $Z I P 7^{-1-}$ cells rescued cell proliferation, compared with transduction with GFP or ZIP7 ${ }^{\text {G178D }}$ (Fig. 3, A and B). WT ZIP7 expression also rescued ER stress, with a decrease in the CHOP nucleus/cytoplasmic ratio, PDI intensity, and PDI area (Fig. 3, C-E) compared with GFP or ZIP7 ${ }^{\text {G178D }}$ transduced cells (Fig. 3, C-E). These results conclusively demonstrate that loss of ZIP7 results in decreased cell proliferation and induction of ER stress.

ZIP7 Is Essential for Regulation of Cytosolic Zinc Levels. The ER is a site of zinc storage, and it has been hypothesized that ZIP7 transports zinc into the cytoplasm from the ER stores (Taylor et al., 2008). However, whether loss of ZIP7 affects ER or cytosolic zinc levels has never been conclusively demonstrated. Given our confirmation of earlier studies demonstrating that ZIP7 is localized to the ER (Fig. 1), we sought to measure zinc concentrations in the ER and cytosol using subcellular fractionation of $\mathrm{ZIP}^{+/+}$and $Z I P 7^{-/-}$cells. Specifically, we measured zinc concentrations in the respective ER and cytosolic fractions using inductively coupled plasma mass spectrometry (ICP-MS). Consistent with previous reports in WT cells (Qin et al., 2011; Sun et al., 2015), we observed that the zinc concentration in the cytoplasmic 
A

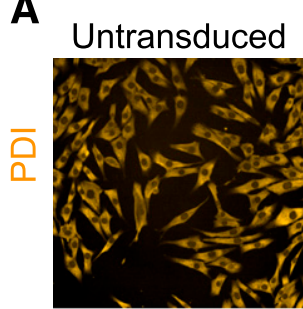
+ WT ZIP7

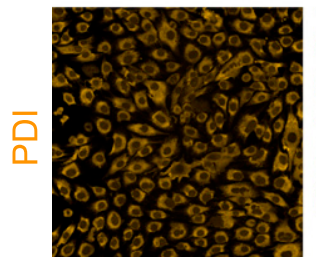

+ GFP

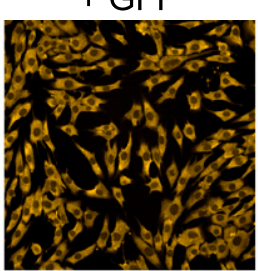

+ G178D

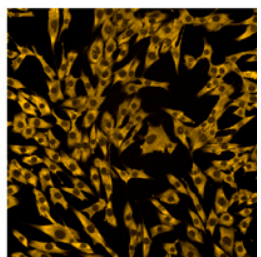

B

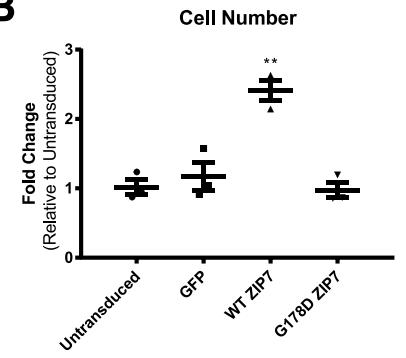

D

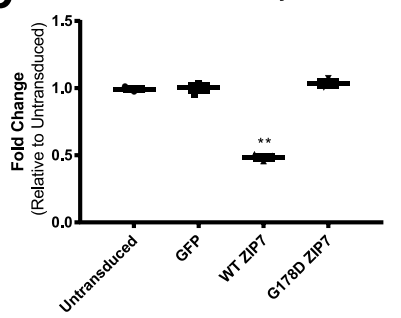

C

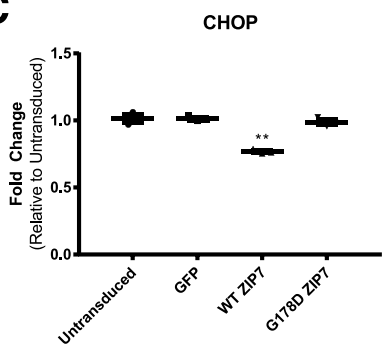

E

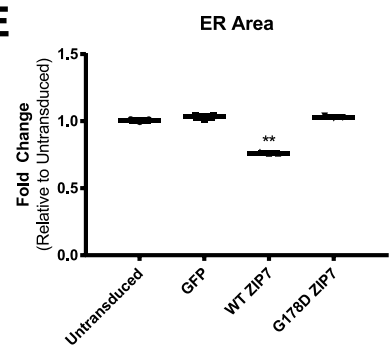

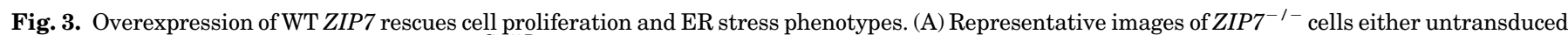

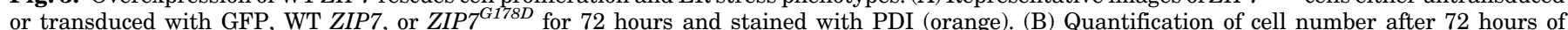

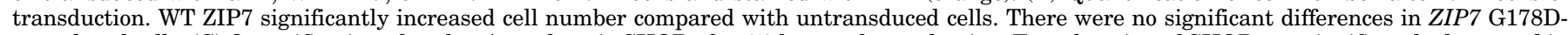

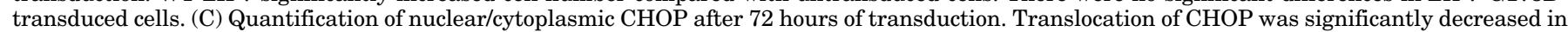

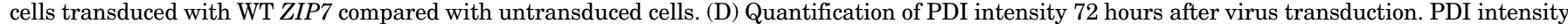

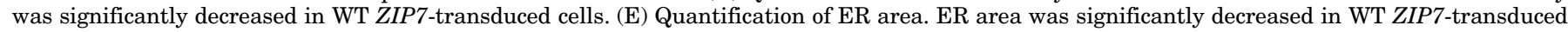

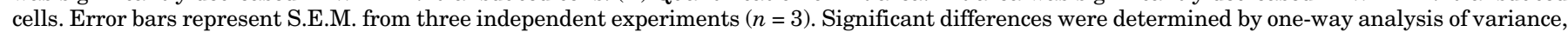
followed by Dunnett's test $(* * P<0.01)$.

fraction $(\sim 1400 \mathrm{ng} / \mathrm{ml})$ was significantly higher than zinc levels in the ER fraction $(\sim 500 \mathrm{ng} / \mathrm{ml})$ (Fig. 4). In contrast, $\mathrm{ZIP7}^{-1-}$ cells exhibited a significant increase of ER zinc concentration and decrease of cytosolic zinc concentration (Fig. 4). These results are consistent with a model whereby ZIP7 transports zinc from the ER into the cytosol, for which loss of ZIP7 results in a shift of the equilibrium toward an increased ER zinc concentration. Therefore, to directly test this hypothesis, we treated cells with pyrithione, an ionophore that transports zinc through the cell membrane. Indeed, when $Z I P 7^{-1-}$ cells were treated with $500 \mathrm{nM}$ pyrithione, cytosolic zinc levels were significantly increased compared with vehicle, nearly reaching the concentration observed in $Z I P 7^{+/+}$cells (Fig. 4). This was presumably due to pyrithione's ability to shuttle the free zinc present in fetal bovine serum (a component of the media) into the cells. We did not observe any changes in ER zinc in these cells, confirming that the increased ER zinc concentration in $Z I P 7^{-/-}$cells results from an inability to transport zinc out of the ER, rather than a cytoplasmic impairment due to the increased concentration of cytosolic zinc (Fig. 4). Therefore, ZIP7 is essential for maintaining both ER and cytosolic zinc levels.

Cell Proliferation and ER Stress Are Rescued by Restoring Cytosolic Zinc. Given our observation that treating $\mathrm{ZIP}^{-/-}$cells with pyrithione can largely restore cytosolic zinc levels, we next asked whether this was sufficient to rescue the cell proliferation and ER stress phenotypes. Importantly, given that pyrithione rescued the abnormal cytosolic, but not ER, concentration of zinc in $Z I P 7^{-/-}$cells (Fig. 4), this also provided us the opportunity to gauge whether the cell proliferation and ER stress phenotypes are driven by cytoplasmic or ER zinc concentrations. $Z I P 7^{-1-}$ cells were treated with pyrithione for 72 hours, followed by quantification of nuclei density, PDI intensity, PDI area, and CHOP
A

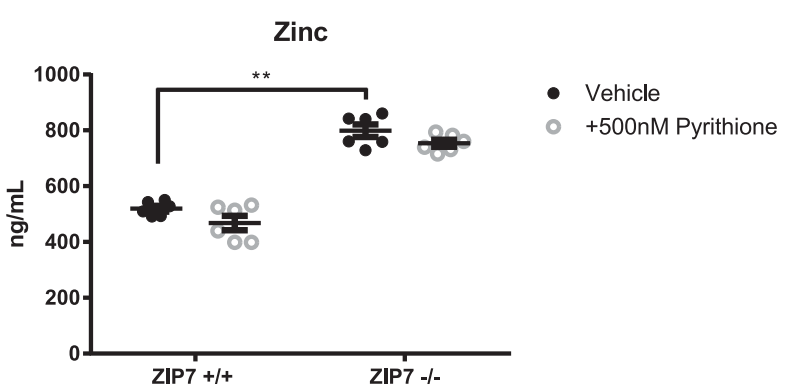

B

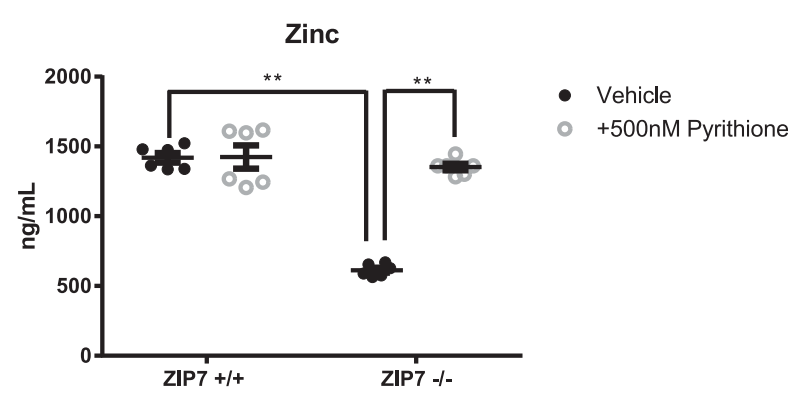

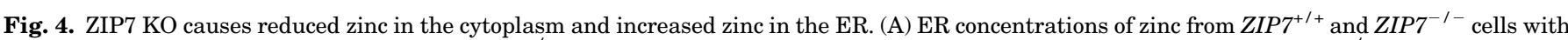

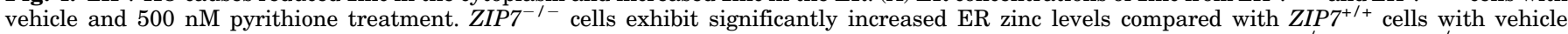

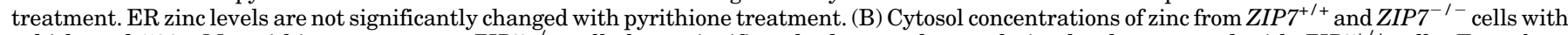

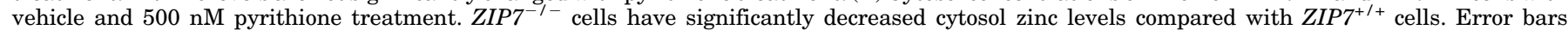
represent S.E.M. $(n=6)$. Significant differences between vehicle- and pyrithione-treated cells were determined by Student's $t$ test $(* * P<0.01)$. 

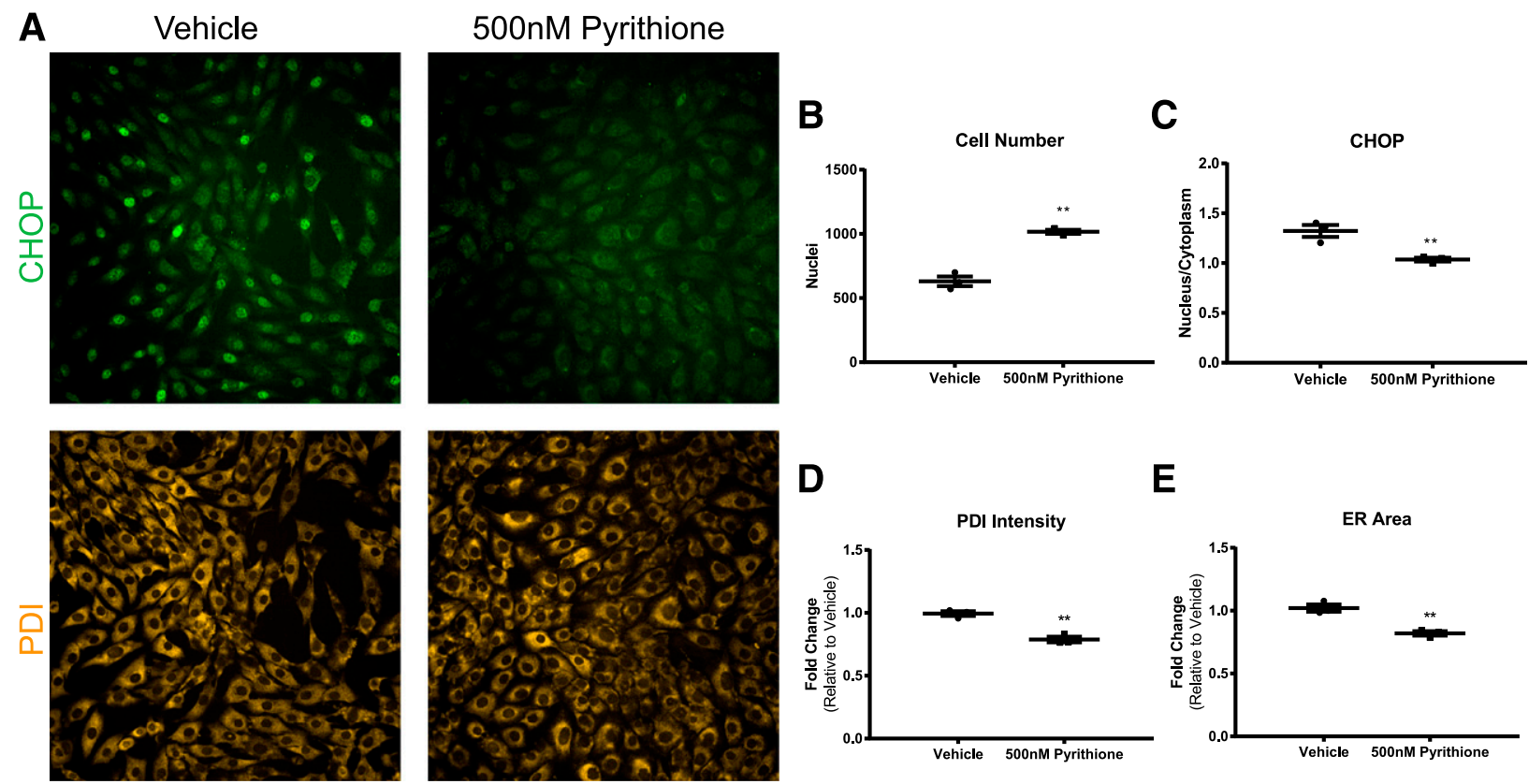

D

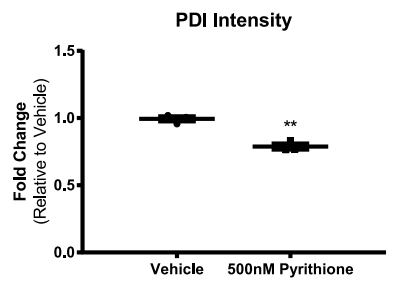

E

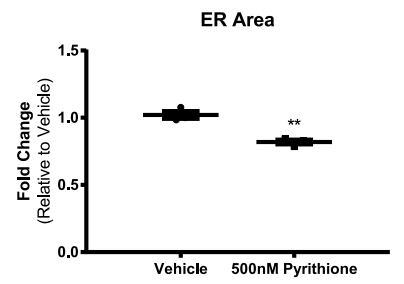

Fig. 5. Pyrithione treatment rescues ER stress and proliferation in $Z I P 7^{-1-}$ cells. (A) Representative images of $Z I P 7^{-1-}$ cells treated with vehicle or pyrithione for 72 hours. Cells were stained with CHOP (green) and PDI (orange). (B) Quantification of cell number in vehicle- and pyrithione-treated cells. (C) Quantification of CHOP in the nucleus versus the cytoplasm in vehicle- and pyrithione-treated cells. (D) Quantification of PDI intensity in vehicle- and pyrithione-treated cells. Values are expressed as fold change relative to vehicle. (E) Quantification of ER area in vehicle- and pyrithione-treated cells. Values are expressed as fold change relative to vehicle. In all graphs, error bars represent S.E.M. from three independent experiments $(n=3)$. Significant differences between vehicle- and pyrithione-treated cells were determined by Student's $t$ test $(* * P<0.01)$.

nuclear/cytoplasmic ratio. Treatment with pyrithione induced significantly increased proliferation within 72 hours (Fig. 5B). Moreover, pyrthione treatment also resulted in significantly decreased PDI intensity, PDI area, and nuclear localization of CHOP (Fig. 5, A and C-E). Together, these findings establish the causality of decreased cytosolic zinc levels in mediating the impaired cell proliferation and induction of ER stress in $Z I P 7^{-1-}$ cells.

Phenotypic Drug Screen in $\mathrm{ZIP7}^{-/-}$Cells. Phenotypic drug screening is a powerful tool to identify compounds that affect a given cellular phenotype. Such screening functions in a target-agnostic manner and has the possibility of uncovering novel biology. We performed a small-scale drug screen with 2816 internal kinase inhibitor compounds at a concentration of $1 \mu \mathrm{M}$ to search for compounds that alleviate ER stress in $Z I P^{-I-}$ cells using the nuclear translocation of CHOP as a readout. We used pyrithione treatment as a positive control and selected compounds with at least 70\% inhibition of CHOP translocation compared with the positive control. Based on these criteria, we identified 33 compound hits. To confirm the hits, we performed a secondary screen in triplicate in which we also quantified cell number, PDI intensity, and PDI area. Of the 33 initial hits, 30 were confirmed to inhibit CHOP translocation when tested in triplicate. Interestingly, we identified one compound (compound A) that significantly inhibited CHOP translocation, increased cell number, and decreased PDI intensity and area (Fig. 6, D-G). We tested compound A in dose response and only found an effect at $1 \mu \mathrm{M}$ (Supplemental Fig. 1). The structure of compound A (4-cyano-N-[2-(4,4-dimethylcyclohex-1-en-1-yl)-4-(2,2,6,6-tetramethyl-1,1-dioxidotetrahydro2H-thiopyran-4-yl)phenyl]-1H-imidazole-2-carboxamide) is shown in Fig. 6C. Compound A was identified internally as an inhibitor of CSF1R with an $\mathrm{IC}_{50}$ of $\sim 3 \mathrm{nM}$ (data not shown). However, compound A is not rescuing the $\mathrm{ZIP}^{-/-}$ phenotypes in a CSF1R-dependent manner because $\mathrm{ZIP7}^{-/-}$ cells do not express CSF1R (data not shown). Additionally, other CSF1R inhibitors were present in the screening library but did not rescue the $\mathrm{ZIP}^{-/-}$phenotypes (data not shown). We also tested compound $\mathrm{A}$ in $Z I P 7^{+/+}$cells and observed that compound A does not have any effect on proliferation or ER stress in $Z I P 7^{+/+}$cells (Supplemental Fig. 2). This result demonstrates that compound $\mathrm{A}$ is specific for rescuing phenotypes associated with loss of ZIP7. To test whether compound A is an inhibitor of other kinases, we performed the Eurofins kinase selectivity panel (1 $\mu \mathrm{M})$ and found that compound A inhibits many other kinases. However, of those kinases, ZIP7 ${ }^{-/-}$cells only express JAK1, PDK1, PLK4, STK16, and TYK2 (Supplemental Table 1). When we tested whether commercially available inhibitors of those kinases could rescue $\mathrm{ZIP}^{-/-}$ phenotypes, none had any significant impact on cell number, CHOP translocation, PDI intensity, or PDI area. Thus, compound $\mathrm{A}$ is rescuing $\mathrm{ZIP} 7^{-/-}$phenotypes through a mechanism other than CSF1R, JAK1, PDK1, PLK4, STK16, or TYK2 inhibition.

Lastly, to examine whether the mechanism of action by which compound A rescued the cellular phenotypes was zincdependent, we treated $Z I P 7^{-1-}$ cells with compound A and measured cellular zinc levels by ICP-MS. We found no significant differences in cytosolic or ER zinc concentrations when cells were treated with compound A (Fig. 7, A and B). This result demonstrates that ER stress and proliferation caused by ZIP7 ablation can be rescued by zinc-independent mechanisms. We also tested whether compound A could block ER stress induced by tunicamycin, brefeldin A, or thapsigargin. We treated $Z I P 7^{+/+}$cells with compound A and found no 
A

2816 Compounds<smiles>CCC</smiles>

33 Primary Hits ( $1 \%$ Hit Rate)<smiles>CCCC</smiles>

30 Confirmed to Inhibit CHOP<smiles>CCCC</smiles>

1 Rescues CHOP, PDI, Proliferation
B
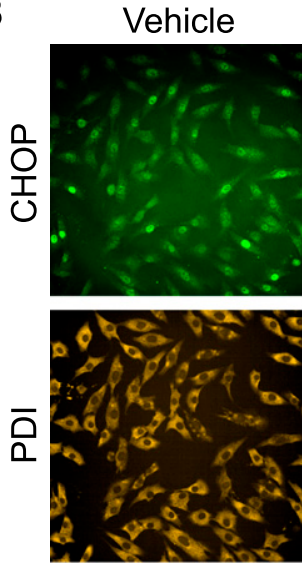

E

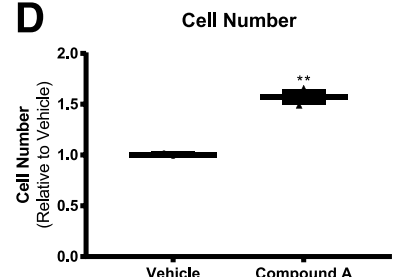

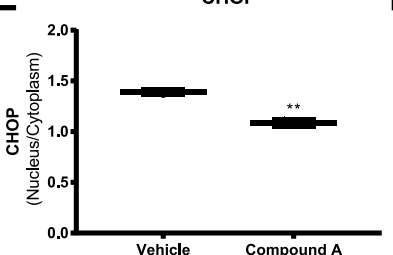
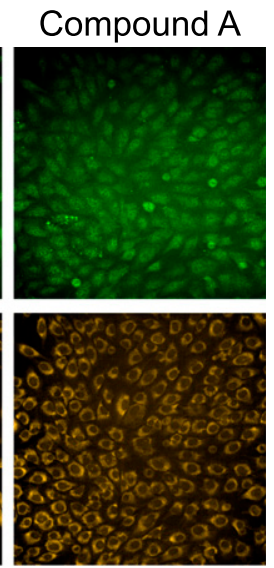

$\mathbf{F}$

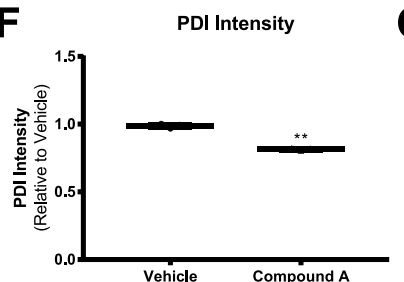

C<smiles>CC1(C)CCC(c2cccc(NC(=O)c3nc(C#N)c[nH]3)c2)CC1</smiles>

4-Cyano-N-[2-(4,4-dimethylcyclohex-1-en-1-yl) -4-(2,2,6,6-tetramethyl-1,1-dioxidotetrahydro$2 \mathrm{H}$-thiopyran-4-yl)phenyl]$1 \mathrm{H}$-imidazole-2-carboxamide

Fig. 6. Phenotypic screen for small molecules that rescue ER stress and proliferation in $Z I P 7^{-1-}$ cells. (A) Schematic of screening results. Two thousand eight hundred and sixteen small molecules were screened at a concentration of $1 \mu \mathrm{M}$. There were 33 primary hits that inhibit CHOP translocation to the nucleus, and 30 of those were confirmed in the secondary screen. One compound was identified to rescue all ZIP7 ${ }^{-1-}$ phenotypes, including cell number, PDI intensity, and ER area. (B) Representative images of vehicle and compounds that rescue CHOP nuclear translocation, PDI intensity, and PDI area. Cells are stained with CHOP (green) and PDI (orange). (C) Structures of compound A. (D) Quantification of cell number in vehicle- and compound-treated cells. (E) Quantification of CHOP in the nucleus versus the cytosol. Values are expressed as relative to vehicle. (F) Quantification of PDI intensity in vehicle- and compound-treated cells. Values are expressed as relative to vehicle. (G) Quantification of ER area in vehicle- and compound-treated cells. Values are expressed as relative to vehicle. Error bars in all graphs represent S.E.M. from three independent experiments $(n=3)$. Significant differences between vehicle- and compound-treated cells were determined by Student's $t$ test $\left({ }^{* *} P<0.01\right)$.

significant differences in the CHOP nucleus/cytoplasmic ratio (Fig. 7C). Treating $Z I P 7^{+/+}$cells with tunicamycin, brefeldin $\mathrm{A}$, and thapsigargin induced CHOP translocation to the nucleus (Fig. 7C). Interestingly, compound A failed to block
CHOP translocation induced by tunicamycin, brefeldin A, or thapsigargin (Fig. 7C). These results demonstrate that compound A may be specific for rescuing ER stress only when ER stress is induced by low cytosolic zinc.
A

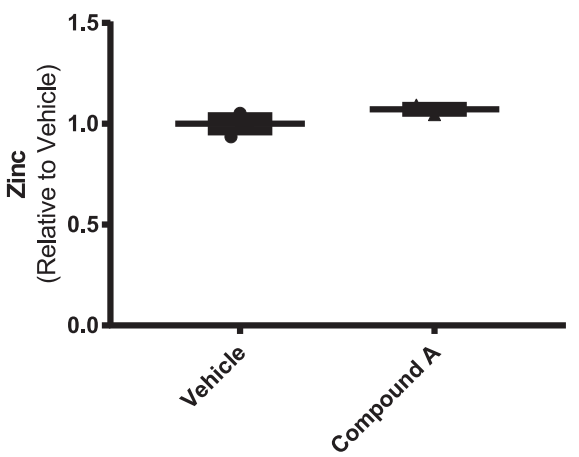

B

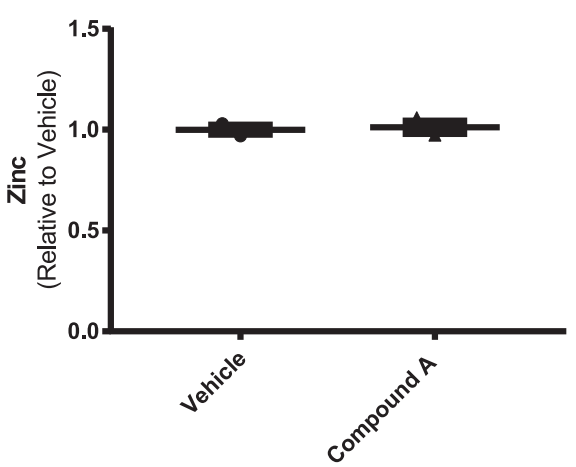

C

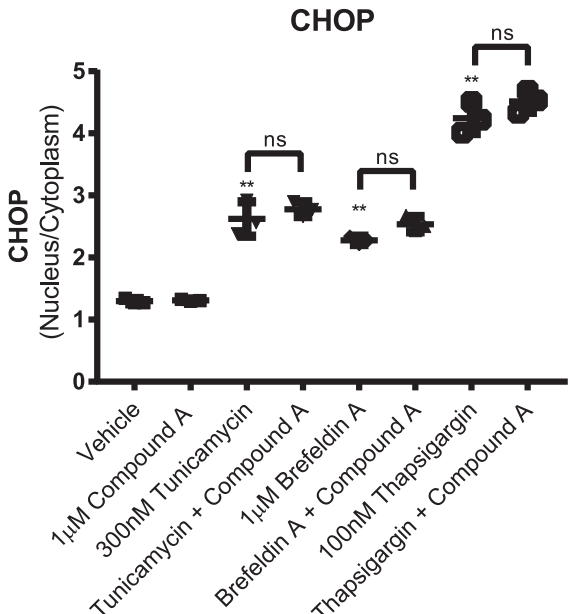

Fig. 7. Compound A rescues $Z I P 7^{-1-}$ phenotypes independent of zinc levels. (A) Quantification of cytosolic zinc concentration by ICP-MS. Compound A does not significantly alter cytosolic zinc levels. (B) Quantification of ER zinc levels by ICP-MS. Compound A does not significantly alter ER zinc levels. Error bars represent S.E.M. from three biologic replicates. (C) Quantification of CHOP in the nucleus versus the cytosol in cells treated with inducers of ER stress. Tunicamycin, brefeldin A, and thapsigargin induce CHOP translocation to the nucleus, and compound A does not block the CHOP translocation. Significant differences between vehicle and ER stress inducer were determined by one-way analysis of variance, followed by Dunnett's test $(* * P<0.01)$. There were no significant differences between ER stress inducer-treated cells versus ER stress inducer and compound A-treated cells. Error bars represent S.E.M. from three independent experiments $(n=3)$. ns, not significant. 


\section{Discussion}

In this study, we investigated the role of ZIP7 in the regulation of cellular zinc levels. We found that CRISPR/Cas9-mediated ablation of ZIP7 results in significantly decreased zinc concentration in the cytosol and increased zinc levels in the ER, with concomitant abnormalities in cell proliferation and ER stress. Both phenotypes are rescued when ZIP7 is overexpressed or when cytosolic zinc levels are restored with pyrithione. Additionally, we identified a small molecule that completely rescues the phenotypic effects of ZIP7 KO by a zinc-independent mechanism, which might have important implications for treating diseases of aberrant zinc homeostasis.

Zinc has a well-established role as a frequent cofactor in enzyme catalysis and protein folding (Andreini et al., 2011; Andreini and Bertini, 2012). More recently, zinc also has a proposed role as a second messenger for intracellular signaling and transduction pathways. Thus, establishing how cytosolic zinc levels are regulated is important for understanding how cells maintain a variety of critical functions. In this study, we definitively demonstrate that ZIP7 is essential for maintenance of cytosolic zinc levels. Even though we observed upregulation of other zinc transporters in $Z I P 7^{-1-}$ cells, these changes were not sufficient to compensate for the loss of ZIP7. This suggests that ZIP7 is obligatory for maintaining cytosolic zinc. Furthermore, we show that insufficient zinc levels in the cytosol impair cell proliferation and induce ER stress. Treatment of $Z I P 7^{-1-}$ cells with pyrithione rescued ER stress phenotypes, but notably only with restoration of cytosolic zinc levels and without any evidence of a restored ER zinc concentration. Previous studies hypothesized that accumulation of zinc in the ER was the cause of ER stress in ZIP7 deficiency (Bin et al., 2017), but based on the selective rescue of cytosolic, but not ER, zinc by pyrithione, our findings indicate that low cytosolic zinc was the driver of both ER stress and proliferation defects. Bin et al. (2017) hypothesized that elevated zinc in the ER causes PDI to aggregate, which prevents the proper folding of proteins and ultimately leads to ER stress. Therefore, although our results demonstrate that low levels of zinc in the cytosol are the cause of ER stress, we cannot rule out that altered activity of PDI might also be a contributing factor.

ZIP7 is one of the $10 \%$ of genes that are consistently upregulated in breast cancer (Hogstrand et al., 2009). Our results suggest that increased expression of ZIP7 could cause growth advantages, possibly by increasing zinc levels in the cytosol. Thus, targeting intracellular zinc levels could be of therapeutic benefit in breast cancer. One way to modulate intracellular zinc would be to identify modulators of ZIP transporters that reside on the cell surface. Our results suggest that intracellular zinc levels need to be carefully fine-tuned as low intracellular zinc causes growth retardation, for which increased cytosolic zinc may lead to a growth advantage.

To date there are numerous reports of mutations in $\mathrm{ZnT}$ and ZIP transporters that cause disease and dozens of singlenucleotide polymorphisms that may contribute to an array of medical conditions. Presumably these mutations are causing aberrant zinc or other metal homeostasis, which highlights the need for therapies that can compensate for changes in zinc transporter activity or function; however, this remains to be systemically evaluated. In our study, we identified a compound that completely rescues the proliferation and ER stress consequences of ZIP7 KO, which validates that disorders of zinc homeostasis can be therapeutically targeted. Moreover, given that the compound that we identified rescues ER stress and proliferation without changing zinc levels, phenotypes caused by aberrant metal homeostasis appear to be possible to rescue by zinc-independent mechanisms. Future studies to elucidate both the zinc-dependent and zincindependent mechanisms hold therapeutic potential for treating disease caused by zinc imbalances.

In conclusion, we propose that ZIP7 is an essential regulator of cytosolic zinc levels and that sufficient levels of zinc in the cytosol are necessary for normal proliferation and normal ER function. ZIP7 could be a therapeutic target for conditions in which zinc levels are dysregulated or in conditions of high ER stress.

\section{Acknowledgments}

We thank Kristopher Standish for support with RNA-seq analysis.

\section{Authorship Contributions}

Participated in research design: Woodruff, Bouwkamp, de Vrij, Lovenberg, Bonaventure, Kushner, Harrington.

Conducted experiments: Woodruff.

Performed data analysis: Woodruff.

Wrote or contributed to the writing of the manuscript: Woodruff, Bonaventure, Kushner, Harrington.

\section{References}

Andreini C and Bertini I (2012) A bioinformatics view of zinc enzymes. J Inorg Biochem 111:150-156.

Andreini C, Bertini I, and Cavallaro G (2011) Minimal functional sites allow a classification of zinc sites in proteins. PLoS One 6:e26325.

Baum AE, Hamshere M, Green E, Cichon S, Rietschel M, Noethen MM, Craddock N, and McMahon FJ (2008) Meta-analysis of two genome-wide association studies of bipolar disorder reveals important points of agreement. Mol Psychiatry 13: 466-467.

Bin BH, Bhin J, Seo J, Kim SY, Lee E, Park K, Choi DH, Takagishi T, Hara T, Hwang $\mathrm{D}$, et al. (2017) Requirement of zinc transporter SLC39A7/ZIP7 for dermal development to fine-tune endoplasmic reticulum function by regulating protein disulfide isomerase. J Invest Dermatol 137:1682-1691.

Boycott KM, Beaulieu CL, Kernohan KD, Gebril OH, Mhanni A, Chudley AE, Redl D, Qin W, Hampson S, Küry S, et al.; Care4Rare Canada Consortium (2015) Autosomal-recessive intellectual disability with cerebellar atrophy syndrome caused by mutation of the manganese and zinc transporter gene SLC39A8. Am $J$ Hum Genet 97:886-893.

Carrera N, Arrojo M, Sanjuán J, Ramos-Ríos R, Paz E, Suárez-Rama JJ, Páramo M, Agra S, Brenlla J, Martínez S, et al. (2012) Association study of nonsynonymous single nucleotide polymorphisms in schizophrenia. Biol Psychiatry 71:169-177.

Fujishiro H, Yano Y, Takada Y, Tanihara M, and Himeno S (2012) Roles of ZIP8, ZIP14, and DMT1 in transport of cadmium and manganese in mouse kidney proximal tubule cells. Metallomics 4:700-708

Gazzellone MJ, Zhou X, Lionel AC, Uddin M, Thiruvahindrapuram B, Liang S, Sun C, Wang J, Zou M, Tammimies K, et al. (2014) Copy number variation in Han Chinese individuals with autism spectrum disorder. J Neurodev Disord 6:34.

Girijashanker K, He L, Soleimani M, Reed JM, Li H, Liu Z, Wang B, Dalton TP and Nebert DW (2008) Slc39a14 gene encodes ZIP14, a metal/bicarbonate symporter: similarities to the ZIP8 transporter. Mol Pharmacol 73:1413-1423.

Giunta C, Elcioglu NH, Albrecht B, Eich G, Chambaz C, Janecke AR, Yeowell H, Weis M, Eyre DR, Kraenzlin M, et al. (2008) Spondylocheiro dysplastic form of the Ehlers-Danlos syndrome-an autosomal-recessive entity caused by mutations in the zinc transporter gene SLC39A13. Am J Hum Genet 82:1290-1305.

Groth C, Sasamura T, Khanna MR, Whitley M, and Fortini ME (2013) Protein trafficking abnormalities in Drosophila tissues with impaired activity of the ZIP7 zinc transporter Catsup. Development 140:3018-3027.

Hogstrand C, Kille P, Nicholson RI, and Taylor KM (2009) Zinc transporters and cancer: a potential role for ZIP7 as a hub for tyrosine kinase activation. Trends Mol Med 15:101-111.

Illig CR, Chen J, Meegalla SK, and Wall MJ (2016) inventors, Janssen Pharmaceutica NV, assignee. Inhibitors of c-fms kinase. U.S. patent US2008/080081. 2009 Apr 23

Kambe T, Tsuji T, Hashimoto A, and Itsumura N (2015) The physiological, biochemical, and molecular roles of zinc transporters in zinc homeostasis and metabolism. Physiol Rev 95:749-784.

Ko HS, Uehara T, and Nomura Y (2002) Role of ubiquilin associated with proteindisulfide isomerase in the endoplasmic reticulum in stress-induced apoptotic cell death. J Biol Chem 277:35386-35392.

Ohashi W, Kimura S, Iwanaga T, Furusawa Y, Irié T, Izumi H, Watanabe T, Hijikata A, Hara T, Ohara O, et al. (2016) Zinc transporter SLC39A7/ZIP7 
promotes intestinal epithelial self-renewal by resolving ER stress. PLoS Genet 12:e1006349.

O’Roak BJ, Deriziotis P, Lee C, Vives L, Schwartz JJ, Girirajan S, Karakoc E, Mackenzie AP, Ng SB, Baker C, et al. (2011) Exome sequencing in sporadic autism spectrum disorders identifies severe de novo mutations. Nat Genet 43:585-589.

Pinilla-Tenas JJ, Sparkman BK, Shawki A, Illing AC, Mitchell CJ, Zhao N, Liuzzi JP, Cousins RJ, Knutson MD, and Mackenzie B (2011) Zip14 is a complex broad-scope metal-ion transporter whose functional properties support roles in the cellular uptake of zinc and nontransferrin-bound iron. Am J Physiol Cell Physiol 301:C862-C871.

Qin Y, Dittmer PJ, Park JG, Jansen KB, and Palmer AE (2011) Measuring steadystate and dynamic endoplasmic reticulum and Golgi $\mathrm{Zn} 2+$ with genetically encoded sensors. Proc Natl Acad Sci USA 108:7351-7356.

Ron D and Habener JF (1992) CHOP, a novel developmentally regulated nuclear protein that dimerizes with transcription factors C/EBP and LAP and functions as a dominant-negative inhibitor of gene transcription. Genes Dev 6:439-453.

Shalem O, Sanjana NE, Hartenian E, Shi X, Scott DA, Mikkelson T, Heckl D, Ebert BL, Root DE, Doench JG, et al. (2014) Genome-scale CRISPR-Cas9 knockout screening in human cells. Science 343:84-87.

Sun Q, Zhong W, Zhang W, Li Q, Sun X, Tan X, Sun X, Dong D, and Zhou Z (2015) Zinc deficiency mediates alcohol-induced apoptotic cell death in the liver of rats through activating ER and mitochondrial cell death pathways. Am J Physiol Gastrointest Liver Physiol 308:G757-G766.
Taylor KM, Morgan HE, Johnson A, and Nicholson RI (2004) Structure-function analysis of HKE4, a member of the new LIV-1 subfamily of zinc transporters. Biochem J 377:131-139.

Taylor KM, Vichova P, Jordan N, Hiscox S, Hendley R, and Nicholson RI (2008) ZIP7mediated intracellular zinc transport contributes to aberrant growth factor signaling in antihormone-resistant breast cancer cells. Endocrinology 149:4912-4920.

Tuschl K, Meyer E, Valdivia LE, Zhao N, Dadswell C, Abdul-Sada A, Hung CY, Simpson MA, Chong WK, Jacques TS, et al. (2016) Mutations in SLC39A14 disrupt manganese homeostasis and cause childhood-onset parkinsonism-dystonia. Nat Commun 7:11601.

Yamasaki S, Sakata-Sogawa K, Hasegawa A, Suzuki T, Kabu K, Sato E, Kurosaki T, Yamashita S, Tokunaga M, Nishida K, et al. (2007) Zinc is a novel intracellular second messenger. J Cell Biol 177:637-645.

Yan G, Zhang Y, Yu J, Yu Y, Zhang F, Zhang Z, Wu A, Yan X, Zhou Y, and Wang F (2012). Slc39a7/zip7 plays a critical role in development and zinc homeostasis in zebrafish. PloS One 7:e42939.

Address correspondence to: Dr. Anthony W. Harrington, Neuroscience Discovery, Janssen Research and Development, 3210 Merryfield Row, San Diego, CA 92121. E-mail: aharrin1@its.jnj.com 